\title{
Flexible Carbon Nanotube Synaptic Transistor for Neurological Electronic Skin Applications
}

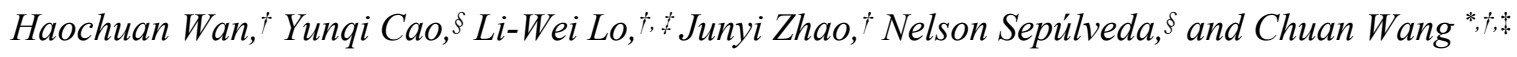

$\dagger$ Electrical and Systems Engineering and Institute of Materials Science and Engineering, Washington University in St. Louis, St Louis, Missouri 63130, United States

$\S$ Electrical and Computer Engineering, Michigan State University, East Lansing, Michigan 48824, United States

*Corresponding author: chuanwang@wustl.edu

\section{Supporting information}




\section{S1. Equations for fitting the curves of synaptic behavior measurements}

\begin{tabular}{ccc}
\hline $\begin{array}{c}\text { Name of the } \\
\text { measurement }\end{array}$ & Equation & $\mathbf{R}^{\mathbf{2}}$ \\
\hline STP (potentiation) & $\Delta \mathrm{PSC}=-0.290+0.0739 \cdot \exp \left(V_{\mathrm{pre}} / 0.843\right)$ & 0.993 \\
STP (depression) & $\Delta \mathrm{PSC}=1.076-0.435 \cdot \exp \left(-V_{\mathrm{pre}} / 0.980\right)$ & 0.998 \\
LTP (potentiation) & $\Delta \mathrm{PSC}=-0.571+4.087 \cdot 10^{-5} \cdot \exp \left(V_{\mathrm{pre}} / 0.345\right)$ & 0.956 \\
LTP (depression) & $\Delta \mathrm{PSC}=0.392-0.174 \cdot \exp \left(-V_{\mathrm{pre}} / 0.841\right)$ & 0.996 \\
PPF & $\mathrm{PPF}$ index $=3.941 \cdot 10^{7} \cdot \exp (-\Delta t / 2.835)+363.1 \cdot \exp (-\Delta t / 45.10)$ & 0.999 \\
STDP (potentiation) & +123.82 & 0.993 \\
STDP (depression) & $\zeta=0.610+2.350 \cdot \exp \left(-\Delta t_{\text {pre-post }} / 6.22\right)$ & 0.990 \\
\hline
\end{tabular}

Table S1. Summary of fitting curve equations in synaptic behavior measurements.

\section{S2. Output curves of the sSWCNT synaptic transistor}

Output curves $\left(I_{\mathrm{DS}}-V_{\mathrm{DS}}\right)$ of the synaptic transistor are shown in Figure $\mathrm{S} 1$ with $V_{\mathrm{GS}}$ ranging from $+4 \mathrm{~V}$ (off) to $-4 \mathrm{~V}$ (on). The curves exhibit clear linear region at low $V_{\mathrm{DS}}$ and saturation region at high $V_{\mathrm{DS}}$, suggesting proper MOSFET-like behavior of the synaptic transistor.



Figure S1. Output characteristics of sSWCNT synaptic transistor. 


\section{S3. Device uniformity}

Five synaptic transistors were measured and their hysteresis curves and synaptic response curves are shown in Figure S2a and Figure S2b. For device \#2 to \#5, with a $V_{\text {pre }}$ of $+4 \mathrm{~V}$ and a pulse width of $10 \mathrm{~ms}$, the synaptic transistors show fairly good uniformity with an average LTP of $20.80 \%$, standard deviation $\delta=$ $0.98 \%$ and average STP of $24.93 \%, \delta=2.48 \%$. This suggests a good controllability of the density and distribution of the interface trap states among different devices during the fabrication processes. PSC curve of device \#1 shows a large deviation from the rest of the devices, which can be attributed to its distinct hysteresis behavior shown in Figure S2a.


Figure S2. (a) Double sweep transfer curves of the synaptic transistors showing the hysteresis of different devices. (b) PSC curves of the corresponding devices with same excitation of $V_{\text {pre }}=+4 \mathrm{~V}$ and pulse width of $10 \mathrm{~ms}$. All measurements are conducted at $\left|V_{\mathrm{DS}}\right|=1.0 \mathrm{~V}$. 


\section{S4. Force measurement using commercial force sensor}

The loading force applied to the FENG is measured by a commercial force sensor (FSR01CE, Ohmite). The force sensor is a resistive device whose resistance decreases with increasing force. The sensor is first calibrated by using standard weights and the calibration curve is shown in Figure S3a. Figure S3b shows the real-time resistance change data of the force sensor during the output signal $V_{\text {OUT }}$ characterization in Figure 6e. The value of the applied force is extracted by averaging the peak resistance during each measurement and fit it into the calibration curve. The peaks from Figure S3b and Figure 6e are highly correlated, suggesting good reliability of the force sensor.

(a)

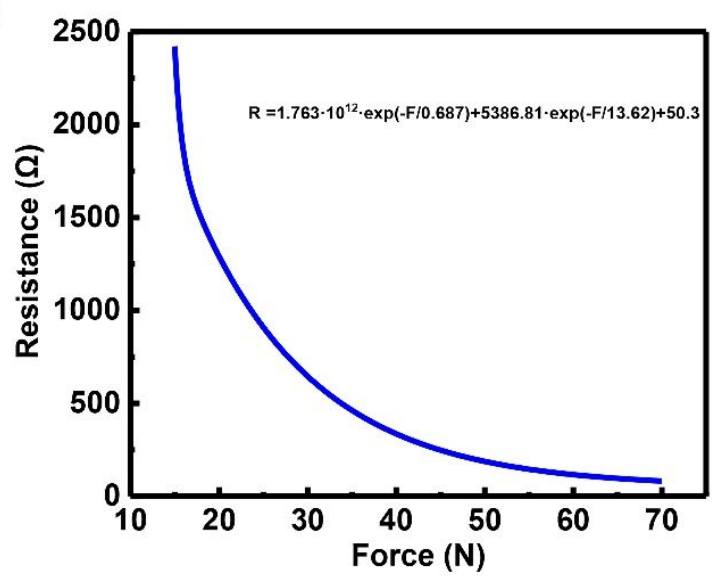

(b)

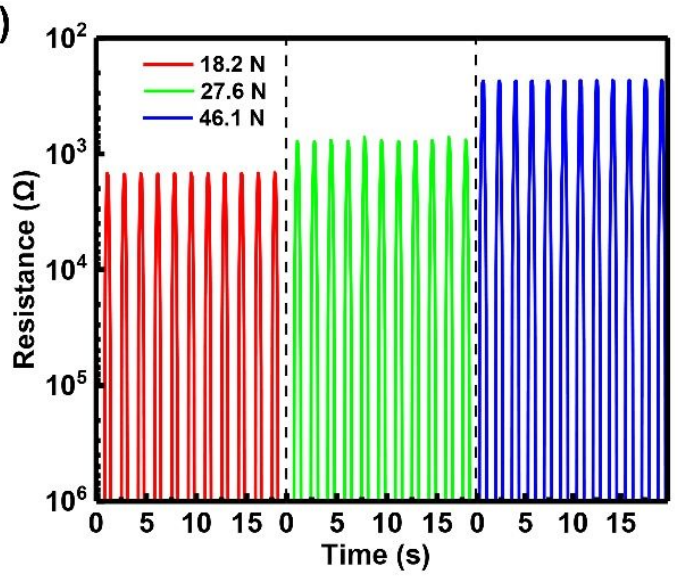

Figure S3. (a) Calibration curve and equation for fitting the Resistance - Force relationship of the commercial force sensor (FSR01CE, Ohmite). (b) Resistance change of the force sensor under periodic loading force and different force amplitude. 